\title{
This is my unit: ICU, University Hospital Centre, Zagreb, Croatia
}

Adriano Friganovic, RN; BsN, Head Nurse, Department of Anesthesiology and Intensive Care of Cardiac Surgery Patients, University Hospital Centre Zagreb

Key Words: Croatia intensive care unit nursing *

E-mail: adriano@hdmsarist.hr

\section{ESPAÑOL}

Eta es mi unidad: UCI, University Hospital Centre, Zagreb, Croacia

Palabras clave

Croacia, enfermería, unidad de cuidado intensivo

Resumen

Este articulo describe la unidad de cuidado intensivo de un topico hospital universitario en Croacia.

\section{SUMMARY}

- This article describes the intensive care unit of a typical university hospital in Croatia.

\section{ABOUT THE HOSPITAL}

The University Hospital Centre Zagreb is the biggest and most important public hospital in Croatia as well as a unique institution regarding many medical procedures, diagnostic methods and therapeutic procedures including highly differentiated procedures of top quality.

The hospital has more than 5,200 employees in total, 3,500 of which are medical staff committed to providing medical and nursing services to their patients. The hospital employs 962 physicians and other medical staff with a university degree and 1,900 nurses and medical technicians.

The main site of our hospital centre ("Rebro") was built in 1942. Since then we have been pursuing developments in all areas of medicine, based upon our own as well as international high standards, which have been accepted in our everyday routine. Many of our staff members have had significant influence in the overall progress of medicine, and some procedures have been performed at University Hospital Centre Zagreb for the first time not only in Croatia but in this part of Europe as well.

Here are some examples: the first cardiac catheterisation in children (1950), CT (1976), the first bone marrow transplantation (1983), the first in vitro fertilization (1983), heart transplantation (1988), liver transplantation from deceased and live donors (1990 and 2001 respectively), endoscopic ultrasound and fine needle aspiration biopsy (2001), apheresis of serum lipoproteins (2002).
Recently, several new procedures have been introduced: MSCT, MR spectroscopy, PET-scan, enteroscopy, endoscopy ultrasound with lymph nodes elastography, interventional neuroradiology, gamma knife radiosurgery, radiofrequency ablation of tumorous tissues, peritonectomy and hyperthermic chemotherapy, laparascopic surgery of kidneys and adrenal glands etc.

First diagnostic and treatment procedures in Croatia in new branches of medicine have been established at University Hospital Centre Zagreb as follows: clinical pharmacology in 1973, electrophysiology in 1977, cognitive neurology in 2006 and metabolic diseases in 2006.

Today we have more than fifty referral centres of excellence acknowledged by the Croatian Ministry of Health and Social Welfare. These centres are aiming at continuous monitoring and improving specific areas of the medical profession and science in Croatia.

University Hospital Centre Zagreb actively cooperates with the world's leading hospital institutions, such as: Harvard University (USA), University Hospital Eppendorf, Hamburg (Germany), University Hospital Centre Munich - locations Großhadern and Innenstadt (Germany), etc.

\section{ABOUT THE UNIT}

The Department of Anesthesiology and Intensive Care of Cardiac Surgery Patients was established in 1985 as a part of clinic for surgery. In 1995, the dDepartment became part of the clinic for cardiac surgery and finally in 2005 it became part of the clinic for anesthesiology, reanimation and intensive care.

The intensive care unit has twelve beds fully equipped with monitors, ventilators, perfusion and infusion pumps, aspirators and oxygen therapy systems. (See pictures 1 and 2.) There are around 700 to 800 patient admissions per year, and the average length of stay for patients is 48 hours. We are admitting all patients after open heart surgery. We have 33 nurses in the intensive care unit, and the nurse to patient ratio is $2: 1$.

The unit has a head nurse and four shift leaders who are educated at least ton bachelor degree level. The head nurse defines processes of nursing care, suggests guidelines and manages human resources. The head nurse is also responsible for nursing education. Since there is no formal education for critical care nurses in Croatia, all nurses who start work in our unit are provided with internal education through six different modules (anatomy of heart, haemodynamic monitoring, blood transfusion, infection control, drugs and mechanical support). Every shift leader has a different role and provide lectures to young nurses.

All nurses in our unit are active members of the Croatian Nurses 
Society of Anesthesia, Reanimation, Intensive Care and Transfusion and actively participate in courses and congresses (see picture 3 ).

Two nurses are instructors for resuscitation and six of them have passed advance life support course. Our wish is that all nurses will complete this course.

Furthermore, an area of our expertise, that we are very proud of, is the surgical treatment of chronic heart failure, a field which we are Croatian leaders in, since 1988. We provide intensive care for the largest number of heart transplantations in our country. Mechanical heart support device implantation (ventricular assist device - VAD) is a procedure which is performed in cooperation with the Department of Cardiac Surgery and it is necessary for adequate chronic heart failure treatment as a 'bridge to transplantation'. For providing better nursing care for the patient with mechanical support we have established a team of four nurses who were trained together with the doctors for all kind of mechanical support (ECMO, RVAD, LVAD). These nurses are assigned to share knowledge with other nurses.

Our intensive care unit also has educational goals and serves as the basic teaching facility not only for medical students within the University of Zagreb Medical School but also for nursing students from the School for Applied Studies.

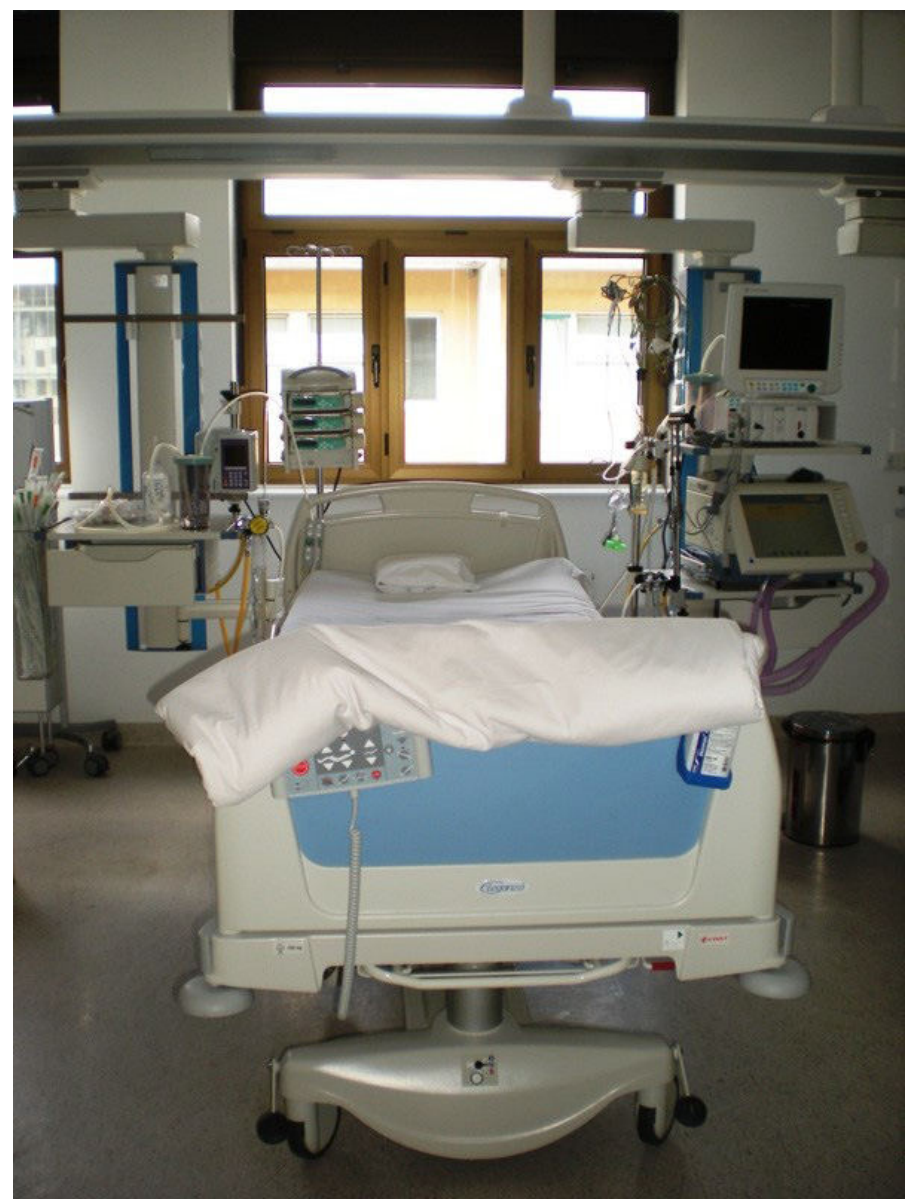

Picture 1. Typical ICU bed space

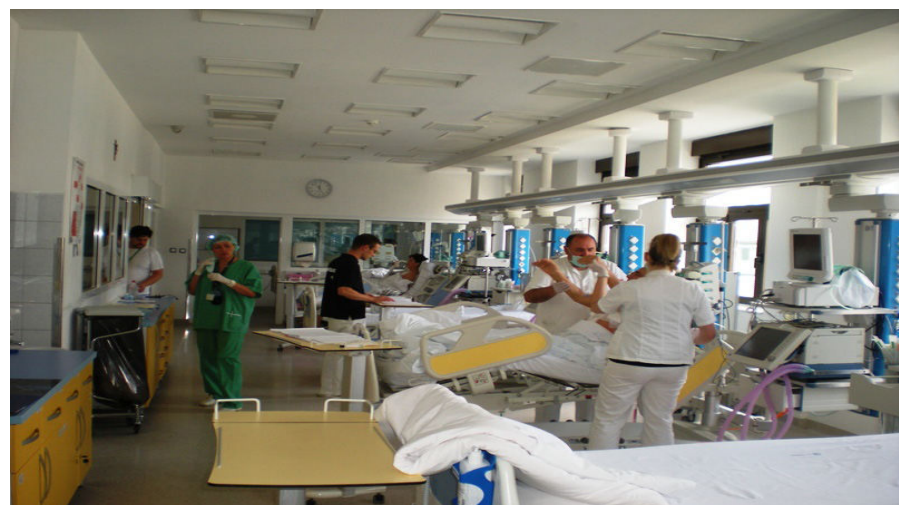

Picture 2. The intensive care unit

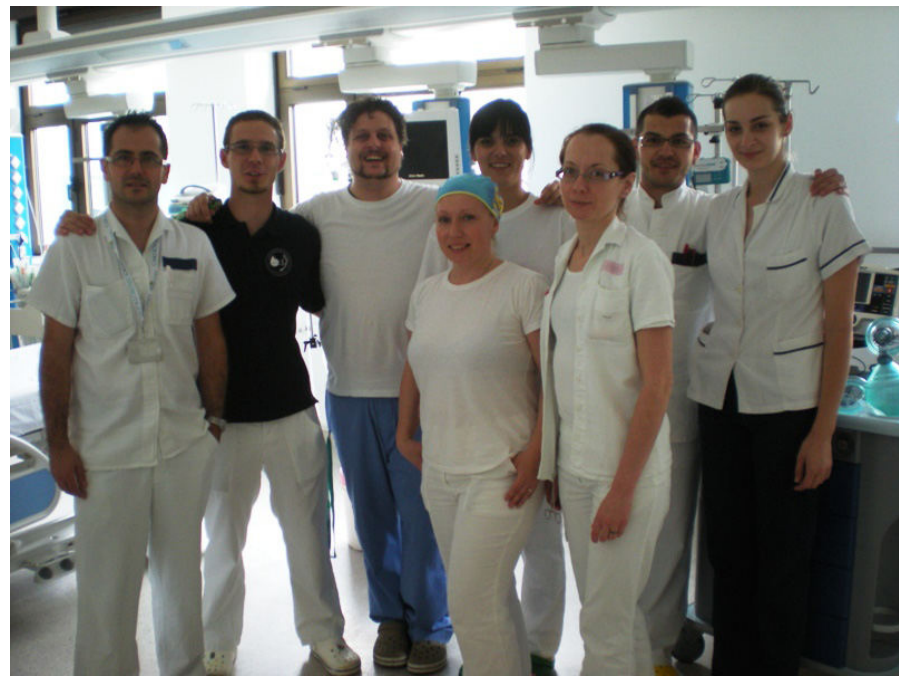

Picture 3. Some of the ICU team members 